\title{
Volatiles Induced from Hypolepis punctata (Dennstaedtiaceae) by Herbivores Attract Sclomina erinacea (Hemiptera: Reduviidae): Clear Evidence of Indirect Defense in Fern
}

\author{
Kerui Huang ${ }^{1,2,3,+}{ }^{\dagger}$, Hui Shang ${ }^{1, *}{ }^{\dagger}$, Qiong Zhou ${ }^{2, *}$, Yun Wang ${ }^{3}$, Hui Shen ${ }^{1}$ and Yuehong Yan ${ }^{1,4}$ (D) \\ 1 Shanghai Chenshan Plant Science Research Center, Chinese Academy of Sciences, Shanghai Chenshan \\ Botanical Garden, Shanghai 201602, China; huangkerui008@163.com (K.H.); shenhui@sibs.ac.cn (H.S.); \\ yhyan@sibs.ac.cn (Y.Y.) \\ 2 College of Life Sciences, Hunan Normal University, Changsha 410081, China \\ 3 College of Life and Environmental Sciences, Hunan University of Arts and Science, Changde 415000, China; \\ wangyun1211@126.com \\ 4 Shenzhen Key Laboratory for Orchid Conservation and Utilization, National Orchid Conservation Center of \\ China and the Orchid Conservation \& Research Center of Shenzhen, Shenzhen 518114, China \\ * Correspondence: shanghui@csnbgsh.cn (H.S.); zhoujoan@hunnu.edu.cn (Q.Z.) \\ + These authors contributed equally to this work.
}

Citation: Huang, K.; Shang, H.; Zhou, Q.; Wang, Y.; Shen, H.; Yan, Y. Volatiles Induced from Hypolepis punctata (Dennstaedtiaceae) by Herbivores Attract Sclomina erinacea (Hemiptera: Reduviidae): Clear Evidence of Indirect Defense in Fern. Insects 2021, 12, 978. https://doi.org/ $10.3390 /$ insects 12110978

Received: 9 October 2021

Accepted: 27 October 2021

Published: 28 October 2021

Publisher's Note: MDPI stays neutra with regard to jurisdictional claims in published maps and institutional affiliations.

Copyright: (c) 2021 by the authors. Licensee MDPI, Basel, Switzerland. This article is an open access article distributed under the terms and conditions of the Creative Commons Attribution (CC BY) license (https:// creativecommons.org/licenses/by/ $4.0 /)$.
Simple Summary: Plants have developed many kinds of indirect defense mechanisms against herbivorous insects, through which the natural enemies are attracted by herbivore-induced plant volatiles to prey on these phytophagous species. There have been many reports regarding this mechanism in spermatophytes, but little is known in ferns. In this study, the relationship between the fern species Hypolepis punctata and the predatory insect Sclomina erinacea was studied. Employing field observations, plant volatile analysis, electrophysiological experiments, and behavioral experiments, we found that not only the plant can attract the assassin bug in the field, but compared with the healthy individuals, the fern being damaged by herbivorous insects also emitted several novel volatile organic compounds, which were strongly attractive to the assassin bug. The results indicate that ferns may also have indirect defense mechanisms using volatile organic compounds, and it is the first report of an indirect defense mechanism in fern.

Abstract: Plants have evolved various self-defense mechanisms against insect feeding. There are many reports regarding both direct and indirect defense mechanisms in seed-plant. However, only direct defenses on ferns were considered and the indirect defense mechanism has never been reported. In this study, it was observed that the fern Hypolepis punctata can attract the assassin bug Sclomina erinacea in the field. We collected and analyzed volatiles from H. punctata healthy individuals and the ones wounded by Bertula hadenalis, using dynamic headspace and GC-MS. We recorded the electroantennogram responses of antennae of $S$. erinacea to different standards of volatile compounds identified from the GC-MS analysis. We also analyzed the behavior of male and female S. erinacea adults in response to volatiles collected from $H$. punctata using a Y-tube olfactometer. The results showed that a number of volatile compounds were produced when the fern was damaged by $B$. hadenalis. Electroantennography and Y-tube olfactometer results showed that some herbivore-induced volatiles and volatiles from undamaged leaves could attract $S$. erinacea. Our research suggests that $H$. punctata can attract insect predators by releasing herbivory-induced volatile organic compounds, and for the first time we found ferns may also have indirect defense mechanisms using volatile organic compounds.

Keywords: electroantennography; GC-MS; Hypolepis punctata; indirect defense; olfactory response; Sclomina erinacea; volatile organic compounds 


\section{Introduction}

The relationship between plants and insects on earth is intricate. Among the approximately one million known insect species, $45 \%$ or more are herbivorous $[1,2]$. To reduce damage from insects, plants have evolved a series of direct or indirect defense mechanisms to prevent insects' predation [3,4]. For direct defense, physically, plants have thorns on the surface and highly lignified tissues, and chemically, they produce various toxic or repellent metabolites against herbivorous insects [5]. The indirect defense is mainly achieved by attracting beneficial insects (such as predators) from additional trophic levels [6]. Being the most common attractant to these predators, volatile organic compounds (VOCs) secreted by plants are mainly terpenes, fatty acid derivatives, and some other aromatic compounds [7-9], they can not only function in the defense process of a single individual, but some can also act as "alarms" to inform other plants to start defense [10-12].

Currently, there are many published pieces of research regarding the mechanism of indirect defense in a seed plant. For instance, after being wounded by Manduca sexta, Nicotiana attenuate can release branched-chain aliphatic acids to attract ants for self-protection [13]. However, for ferns, the focus has been always on direct defense mechanisms. For example, Huang et al. [14] tested extracts from five different parts of ferns and found that they are insecticidal. Markham et al. (2006) showed that proteins from three out of 17 fern species tested have significant toxicity against insects. However, little is known about indirect defense mechanisms in ferns [15].

Using a Gas Chromatography-Mass Spectrometer, Imbiscuso et al. analyzed the composition of volatiles from Pteris vittata after it was attacked by Spodoptera litura and identified three volatile compounds induced after the feeding, including a terpenoid, suggesting that ferns may also employ indirect defense strategies [16]. However, the research did not delve further into behavioral and electrophysiological experiments using the predators of S. litura. Koptur et al. (1998) analyzed relationships between ants and Polypodium plebeium in Mexico [17] and found that the damage to the fern by herbivorous insects was significantly reduced only when the fern was producing nectar and ants were present, suggesting that the fern attracts ants (Formicidae) by secreting nectar to protect them from other insects. Although P. plebeium attracted ants by secreting nectar, this is unlike plants producing volatile compounds for secondary defense attraction mechanisms, because ants were directly benefited from the nectar. In contrast, the volatiles that attract natural enemies do not provide direct benefit to natural enemies during the indirect defense. Therefore, more studies are needed to elucidate the indirect defense mechanisms in ferns.

Natural enemies play essential roles in plant indirect defense. Sclomina erinacea, Hemiptera, Reduviidae is a generalist natural enemy of insects [18] and is mainly distributed in the southeast of China [19]. Hypolepis punctata (Dennstaedtiaceae) also grows in the southeast of China, mainly in tropical and subtropical regions, like Hainan, Fujian, Taiwan, Guangdong, Guizhou, Yunnan, Sichuan, Jiangxi, Zhejiang, and Anhui Provinces. It grows in warm, wet environments and can be found in valleys, along the bank of streams, and at the forest edge [20]. We conducted preliminary field studies in Zhangjiajie, Hunan, and Daweishan, Hunan, in 2013, which indicated that S. erinacea feeds on the larvae of noctuid moths, including Callopistria japonica and Bertula hadenalis (Lepidoptera, Noctuidae). In addition, it also hunts for aphids (Aphidoidea), Drosophila spp., and other agricultural pests. More interestingly, we found that S. erinacea prefers to land on H. punctata to hunt Lepidoptera larvae that feed on ferns, such as the larvae of Callopistria japonica, and rarely on other ferns. It was also notable that the scent of H. punctata, sensed by a human nose, is more discernible than that of many other ferns. Terpenoids are the main attractants to natural enemies in many plants $[2,9,21]$, and in an early study, Hayashi et al. (1977) extracted sesquiterpenes from $H$. punctata [22]. Thus, this characteristic, together with our preliminary field observations, raises the following questions: (1) does H. punctata produce attractant volatiles to attract $S$. erinacea to prevent further herbivory from Lepidoptera insects after initial wounding, and (2) if yes, what are the attractive compounds? 
To answer these questions, the volatiles from healthy and wounded H. punctata (damaged by larvae of noctuid) were compared using GC-MS and determined the electroantennogram (EAG) responses of antennae of S. erinacea to different standards of volatile compounds identified from the GC-MS analysis. The behavior of male and female adults of $S$. erinacea to these volatile compounds was also investigated using a Y-tube olfactometer. Our findings provide insights into the mechanism underlying the attraction of $S$. erinacea to H. punctata.

\section{Materials and Methods}

\subsection{Field Survey of Visitation}

A field survey of the visitation and the behavior of $S$. erinacea had been done in an $H$. punctata population, in a valley in southern Hunan province, the H. punctata individuals were classified judging by the appearance of the leave into two groups: 41 healthy individuals (HI) and 34 damaged individuals (DI, meaning damaged by phytophagous insects). The S. erinacea usually stays on $H$. punctata for hours to a whole day during one visitation, thus the number of $S$. erinacea on each $H$. punctata individual was recorded in the morning. The observation lasted for seven days.

\subsection{Collecting and Analyzing Volatile Organic Compounds from Hypolepis punctata}

VOCs were sampled in naturally growing Hypolepis punctata populations in Fengluanxi $\left(110^{\circ} 7^{\prime} 36^{\prime \prime} \mathrm{N}, 2^{\circ} 19^{\prime} 50^{\prime \prime} \mathrm{E}\right)$, Hunan Province. Several individuals of H. punctata with similar growth status and size that showed no sign of insect damage were selected, and the larvae of Bertula hadenalis, a species of noctuid were selected as the herbivorous insect. We divided all $H$. punctata individuals into three groups: healthy individuals (HI), undamaged individuals that were growing close to damaged individuals (UICD), and damaged individuals (DI, meaning damaged by B. hadenalis). Prior to volatile sampling, one leaf from each individual of Hypolepis punctata was safely enclosed in a fine mesh nylon bag. For the DI group, we added two to three two-instar larvae of Bertula hadenalis into each bag, and for the HI group, no larvae were placed into the bags. Additionally, the HI group individuals were more than $10 \mathrm{~m}$ away from the plants included in the DI group. The individuals of the UICD group were similar to the HI group, as no larvae were added to the bags, but the distance between the UICD individuals and the DI individuals was less than two meters. The mesh bags remained affixed to the leaves for $24 \mathrm{~h}$ before volatile sampling. During VOC sampling, the mesh bags and larvae were removed, and each leaf was enclosed in a Nalophan bag (Toppits, Minden, German) with openings at both ends. One end was fastened securely to the fern stipe together with a glass tube (diameter $4 \mathrm{~mm}$, length $15 \mathrm{~cm}$ ) filled with activated charcoal, and the other end was connected to another glass tube (diameter $4 \mathrm{~mm}$, length $10 \mathrm{~cm}$ ) containing $30 \mathrm{mg}$ adsorbent (Porapak Q). The end of the adsorbent tube was connected to an airflow meter (model LZB-3W, 100-1000 mL/min, Shanghai Hange Biosciences Corporation, Shanghai), which was connected to an air pump (model QC-1S, Beijing Municipal Institute of Labor Protection, Beijing) set to a flow rate of $400 \mathrm{~mL} / \mathrm{min}$ for four hours. Each part mentioned was connected using laboratory-grade PVC tubing. In the negative control experiments, the Nalophan bags were empty. In each location, there were four repeats of the experimental groups (DI, HI, and UICD) and two of the negative control group, and tests were conducted concurrently from 9:00 am to 2:00 pm. Compounds were eluted from the adsorbent tube with $600 \mu \mathrm{L}$ of $\mathrm{n}$-hexane and stored at $-20{ }^{\circ} \mathrm{C}$ until analyzed.

$0.4 \mu \mathrm{L}$ of the eluent was analyzed using a Shimadzu GC-MS (GCMS-QP2010) with a RESTEK Rtx ${ }^{\circledR}-5 \mathrm{MS}$ column $(30 \mathrm{~m} \times 200 \mathrm{~mm}$, film thickness $0.25 \mathrm{~mm})$. The carrier gas used was helium with a flow rate of $2.0 \mathrm{~mL} / \mathrm{min}$. The GC-MS was programmed as follows: injector $250{ }^{\circ} \mathrm{C}$, initial column temperature held at $50{ }^{\circ} \mathrm{C}$ for $3 \mathrm{~min}$, increased at $10{ }^{\circ} \mathrm{C}$ $\mathrm{min}^{-1}$ to $210^{\circ} \mathrm{C}$, maintained for $1 \mathrm{~min}$, increased at $20^{\circ} \mathrm{C} \mathrm{min}{ }^{-1}$ to $250{ }^{\circ} \mathrm{C}$, and held for $3 \mathrm{~min}$. 
We used mass spectra from MS libraries (NIST05, NIST05s, and WILEY7) for tentative peak identification, and only those compounds with a higher concentration than in the control samples were considered. Retention indexes, which were used to convert retention times into system-independent constants, were taken from studies that used the Rtx ${ }^{\circledR}$ 5MS column, as well as those that used the similar HP-5MS column. We compared the composition of VOCs in the individuals by calculating proportions of the contribution of identified compounds, which in each individual were calculated by dividing the area of each peak by the total area of all the peaks, except the peaks of contaminants, and multiplying by 100 .

\subsection{Electroantennogram Recordings}

The responses of antennae of both male and female Sclomina erinacea to certain volatile compounds of Hypolepis punctata were tested using electroantennogram recordings (EAG). Nymphs of Sclomina erinacea were collected from Fengluanxi (110 $7^{\prime} 36^{\prime \prime}$ N, 29 $\left.9^{\circ} 19^{\prime} 50^{\prime \prime} \mathrm{E}\right)$, Hunan Province, and grown to adults under laboratory conditions fed with the larvae of Tenebrio molitor $\left(25^{\circ} \mathrm{C}, 60 \% \mathrm{RH}\right)$. The compounds used for testing (five to be herbivory induced compounds and two green leaf volatiles) were selected according to the results obtained in the VOCs analysis, all the compounds used in this experiment are listed in Table 1. In this experiment, an entire antenna was carefully removed from the head of living adult Sclomina erinacea; 4/5 the length of the scape and 1/10 the length of the flagellum was also removed, and both sides of the antenna were attached to electrodes covered with electrode gel (Spectra 360, Parker Labs, Newark, NJ, USA). Ten $\mu$ L of hexane (as control) or a hexane solution composed of one of the tested seven compounds $(10 \mu \mathrm{g} / \mu \mathrm{L}, 100 \mu \mathrm{g})$ was dotted on a piece of filter paper $(5 \mathrm{~mm} \times 40 \mathrm{~mm})$, which was then inserted into a glass Pasteur pipette. The tip of the pipette was inserted about $4 \mathrm{~mm}$ into a small hole in the wall of a glass tube, into which the antenna with the electrodes was inserted $3 \mathrm{~cm}$ from the open end of the tube without touching the inner wall. A constant airflow $(1 \mathrm{~L} / \mathrm{min})$, passing through the glass tube and over the antenna, was generated and controlled by a Syntech CS-55 air stimulus controller, which also controlled the airflow through the pipette. During scent stimulation, an airflow of $40 \mathrm{~mL} / \mathrm{min}$ was pumped through the Pasteur pipette into the glass tube containing an antenna for $1 \mathrm{~s}$. Each antenna was stimulated two or three times with each compound individually at 60-s intervals, and each compound was repeated using eight different antennae from each sex (male and female). Hexanal was used as the reference standard, which we applied to stimulate the antennae at the beginning and end of each stimulation to correct for the loss of antennal sensitivity. For the correction, we assumed that the decrease of the antennal sensitivity had a linear relationship with time; therefore, data were normalized as follows:

$$
\mathrm{rEAG}=\frac{\mathrm{EAG}(\mathrm{A})}{\mathrm{EAG}(\mathrm{std} 1)+\frac{\mathrm{EAG}(\mathrm{std} 2)-\mathrm{EAG}(\mathrm{std} 1)}{\mathrm{RT}(\mathrm{std} 2)-\mathrm{RT}(\mathrm{std} 1)} \times(\mathrm{RT}(\mathrm{A})-\mathrm{RT}(\mathrm{std} 1))}
$$

where rEAG stands for relative EAG response; EAG(A) stands for the amplitude (mV) of the EAG response to compound A; EAG(std1) stands for the EAG response to the reference standard at the beginning of each stimulation; EAG(std2) stands for the EAG response to the reference standard at the end of each stimulation; RT(A) stands for the time when the stimulation was done with compound A; RT(std1) stands for the start time of the stimulation using the reference standard at the beginning of each stimulation; RT(std2) stands for the end time of the stimulation using the reference standard. 
Table 1. Standard compounds used for the EAG recordings and Y-olfactometer experiment.

\begin{tabular}{ccc}
\hline Compounds & Purity & Source \\
\hline Linalool & $97 \%$ & AccuStandard $^{\circledR}$ \\
1-Octen-3-ol & $98 \%$ & AccuStandard $^{\circledR}$ \\
Hexanal & $97 \%$ & Dr. Ehrenstorfer GmbH \\
trans-2-Hexen-1-al & $98 \%$ & Sigma-Aldrich \\
Nonanal & $98 \%$ & Dr. Ehrenstorfer GmbH \\
$\beta$-Myrcene & $95 \%$ & Sigma-Aldrich \\
$\alpha$-pinene & $98 \%$ & Dr. Ehrenstorfer GmbH \\
\hline
\end{tabular}

\subsection{Y-Olfactometer Experiment}

Olfactory behavior of Sclomina erinacea towards seven different volatile organic compounds (Table 1) produced by Hypolepis punctata was tested using a glass Y-tube olfactometer, and an additional file shows a sample graph of the whole device (Supplementary Materials, Figure S1). Two pear-shaped glass bottles where the odor compounds were placed were connected to each end of the arms of the Y-tube (ID $25 \mathrm{~mm}$; stem $6 \mathrm{~cm}$; arms $15 \mathrm{~cm}$ at a $142^{\circ}$ angle to the stem). Both the Y-tube and the pear-shaped glass bottles were put inside a top opening square box (length $50 \mathrm{~cm}$; width $35 \mathrm{~cm}$; height $12 \mathrm{~cm}$ ), made of paper and painted white to reduce environmental disturbance. The contents of the box were illuminated by three $30 \mathrm{~W}$ fluorescent lamps. Air cleaned by active charcoal and humidified by passing through distilled water was pumped through each pear-shaped bottle into each arm at a rate of $400 \mathrm{~mL} / \mathrm{min}$ using an air pump (model QC-1S, Beijing Municipal Institute of Labor Protection, Beijing). Cuboid agar blocks (2\%; $1.5 \mathrm{~cm}$ length; $1.5 \mathrm{~cm}$ width; $0.5 \mathrm{~cm}$ height) placed in the glass bottles were used to carry each compound and release them into the Y-tube. Each compound was diluted in hexane. For each test, $5 \mu \mathrm{L}$ of the solution $(10 \mu \mathrm{g} / \mu \mathrm{L}, 50 \mu \mathrm{g})$ was injected into one agar block, which was then placed into one of the two pear-shaped glass bottles. Another pear-shaped glass bottle holding an agar block containing $5 \mu \mathrm{L}$ of hexane was used as the control group. Then, one adult insect was released from the opening of the stem into the Y-tube. Choices were scored when adult insects went $2 \mathrm{~cm}$ from the middle of the Y-tube into one arm, and an additional picture shows the position of an adult when it just reached the score point (Supplementary Materials, Figure S2). If an insect did not make any choice after $10 \mathrm{~min}$, then the test was repeated using another insect individual until a choice was finally made. Each compound was tested using at least seven adults of each sex, and each test was repeated three times. To reduce the disturbance of uncertain factors, the Y-tube was cleaned once every five tests using absolute ethanol, and the position of the experimental group and the control group was also exchanged.

\subsection{Data Analysis}

Data of the field study, VOCs proportions, EAG responses, and the Y-tube tests were analyzed using software SPSS 19.0. G-test was used to analyze the average relative VOCs amounts, Duncan's multiple range test was used to compare EAG responses triggered by different compounds and G-test was used to compare the selection of different groups in the field study and the Y-tube test.

\section{Results}

\subsection{The Visitation Observation}

The visitation preference difference of $S$. erinacea individuals between the healthy and the damaged H. punctata in the field observation is shown in Table 2, which demonstrate that, during the seven days of field observations, $S$. erinacea individuals had a strong preference for the damaged Hypolepis punctata $(p<0.05)$ by herbivory for five days in the total seven days, indicating that the herbivory of phytophagous insects on H. punctata can affect the behavior of the assassin bug $S$. erinacea. 
Table 2. The visitation preference difference of Sclomina erinacea individuals between the healthy and the damaged Hypolepis punctata in the field observation for 7 days.

\begin{tabular}{ccccccccc}
\hline & Day 1 & Day 2 & Day 3 & Day 4 & Day 5 & Day 6 & Day 7 & Total \\
\hline $\begin{array}{c}\text { Damaged } \\
\text { individuals }\end{array}$ & 27 & 30 & 26 & 31 & 19 & 18 & 16 & 167 \\
$\begin{array}{c}\text { Healthy } \\
\text { individuals } \\
p \text {-Value }\end{array}$ & 15 & 11 & 9 & 5 & 6 & 7 & 4 & 57 \\
\hline
\end{tabular}

The $p$ Values were generated using G-test for the comparison of the Sclomina erinacea individuals' visitation preference difference between the healthy and the damaged Hypolepis punctata, of which the individual numbers were 34 and 41 respectively.

\subsection{VOCs of Herbivore Damaged and Undamaged Hypolepis punctata}

Table 3 presents the relative percentages of each volatile organic compound collected from healthy individuals (HI), undamaged individuals that are close to damaged individuals (UICD), and damaged individuals (DI, meaning damaged by Bertula hadenalis) of Hypolepis punctata. Twenty-five VOCs were detected in total, with 15 from the HI group, 12 from the UICD group, and 11 from the DI group. The compounds shared among all three groups were $\alpha$-pinene, 1-octen-3-ol, and nonanal. Six terpenes were detected: $\alpha$ pinene, $\beta$-myrcene, sabinene, $\alpha$-farnesene, zingiberene, and linalool. Figure 1 illustrates the comparison of relative percentages of nine VOCs from damaged and undamaged Hypolepis punctata. The relative percentages (divided by the total area of all the peaks) of five of the nine VOCs released from damaged individuals were greater than those released from healthy individuals, significantly for three of them ( $\beta$-myrcene, linalool, $\alpha$-pinene, and nonanal, $p<0.05)$, marginally significantly for $\alpha$-pinene $(p=0.0565)$, and insignificantly for 1-octen-3-o $(p>0.05)$. Thus, these five compounds ( $\beta$-myrcene, linalool, $\alpha$-pinene, nonanal, and 1-octen-3-ol) were assumed to be herbivory-induced volatiles and were selected for the following test in this study.

The other four compounds (hexanal, trans-2-hexenal, 3-hexen-1-ol, and 1-hexanol) are green leaf volatiles (GLVs), and the relative amounts of these four compounds present the form opposite to that of the assumed herbivory induced volatiles. Figure 2 shows the relative percentages of these four GLVs sampled from individuals of the HI, UICD, and DI groups. The individuals of the HI group emitted the most substantial amount of these GLVs, which were released in smaller amounts by individuals of the UICD group, and were the lowest emitted from individuals of the DI group. This indicates that after $H$. punctata were damaged by B. hadenalis larvae, the release of some GLVs was reduced.

\subsection{Electroantennogram Recordings}

Figure 3 illustrates the relative EAG responses of the antennae taken from Sclomina erinacea to tested compounds. The antennae of both male and female S. erinacea shows the highest responses to trans-2-hexenal compared with other tested chemicals $(p<0.05)$. The elicited relative EAG responses of the male antennae in descending order are trans2-hexenal, 1-octen-3-ol, nonanal, linalool, hexanal, $\beta$-myrcene, $\alpha$-pinene, and hexane. For antennae removed from female insects, the EAG responses in descending order are trans-2-hexenal, nonanal, hexanal, 1-octen-3-ol, linalool, $\beta$-myrcene, hexane, and $\alpha$-pinene. All chemicals except for $\alpha$-pinene and $\beta$-myrcene elicit significantly higher relative responses in both male and female antennae than hexane $(p<0.05)$. Linalool, $\alpha$-pinene, trans-2-hexenal, and 1-octen-3-ol elicit higher relative responses in males than in females, and response to 1 -octen-3-ol is significantly different $(p<0.05)$. The other three compounds elicit responses that are higher in females than in males $(p>0.05)$. 
Table 3. Relative percentage of VOCs recorded from the individuals of healthy and Hypolepis punctata damaged by herbivory.

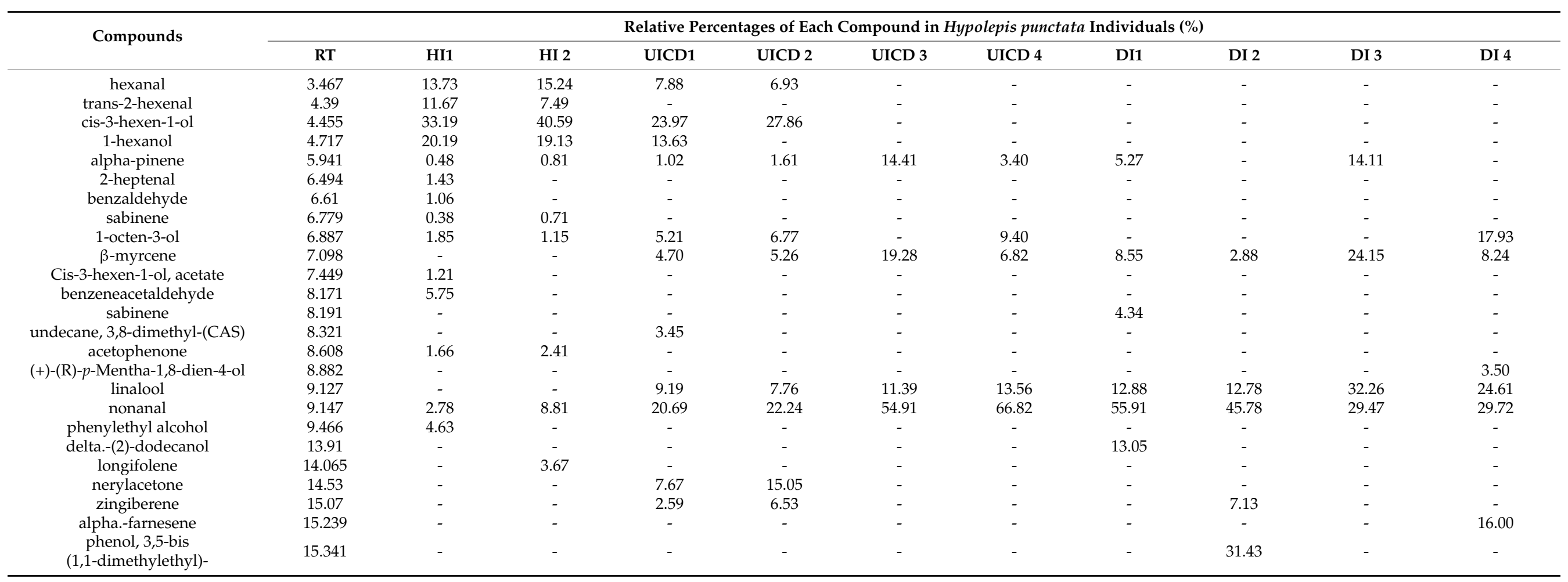

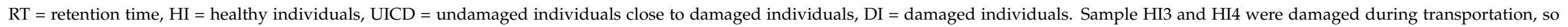
data is missing. 


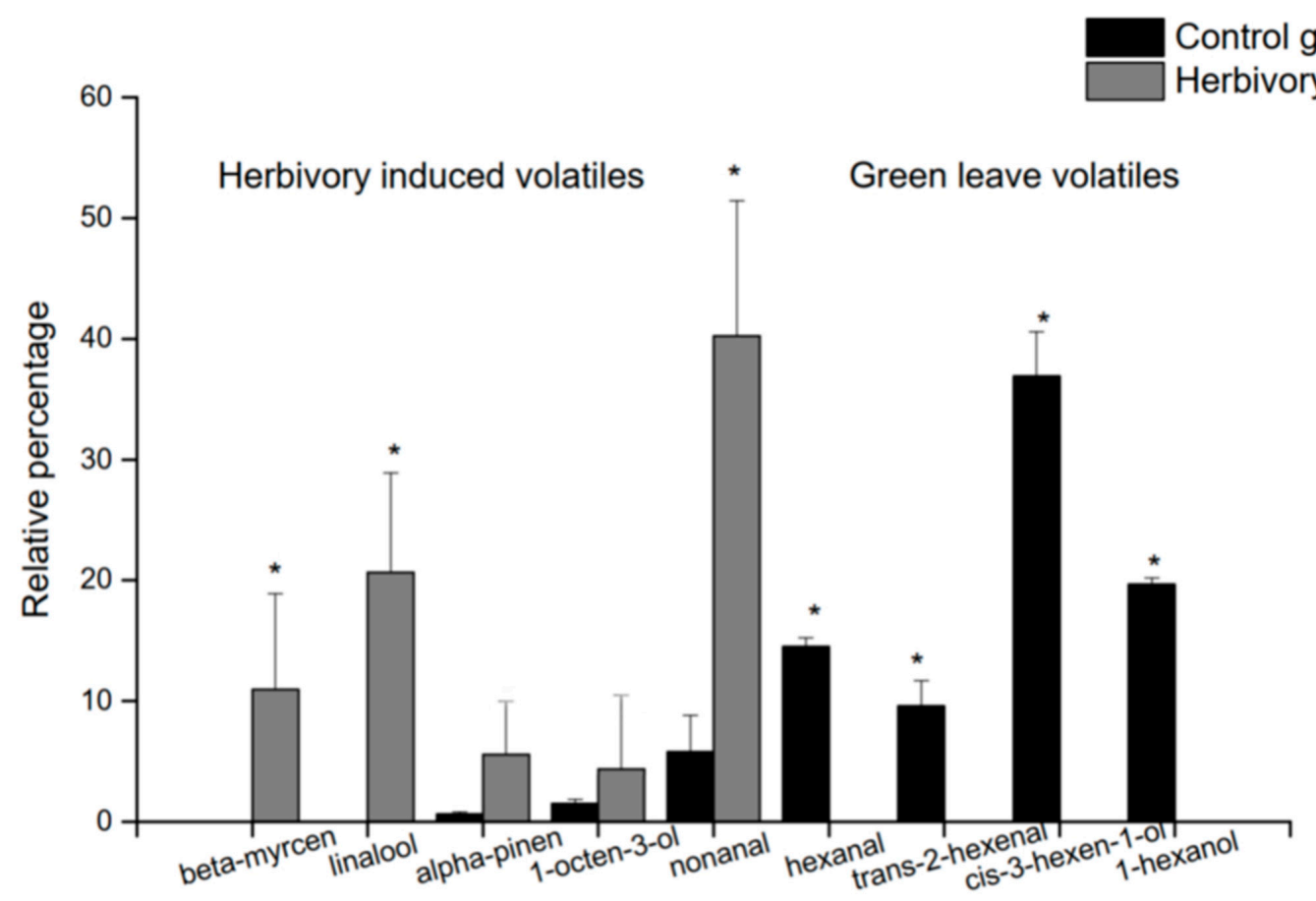

Figure 1. Relative Percentages of Nine VOCs from Damaged and Undamaged Hypolepis punctata. The Y-axis is the average relative percentage of the same compound collected from Hypolepis punctata individuals of the damaged group and undamaged group; each group is represented with a unique color. The $\mathrm{X}$-axis indicates nine chemical compounds; the five on the left side of the vertical line are assumed herbivory induced compounds, and the four on the right side are green leaf volatiles. ${ }^{*}$ indicates that there is a significant difference between the relative percentage of the same compound collected from the control group (HI group) and herbivory group (DI group) $(p<0.05)$.

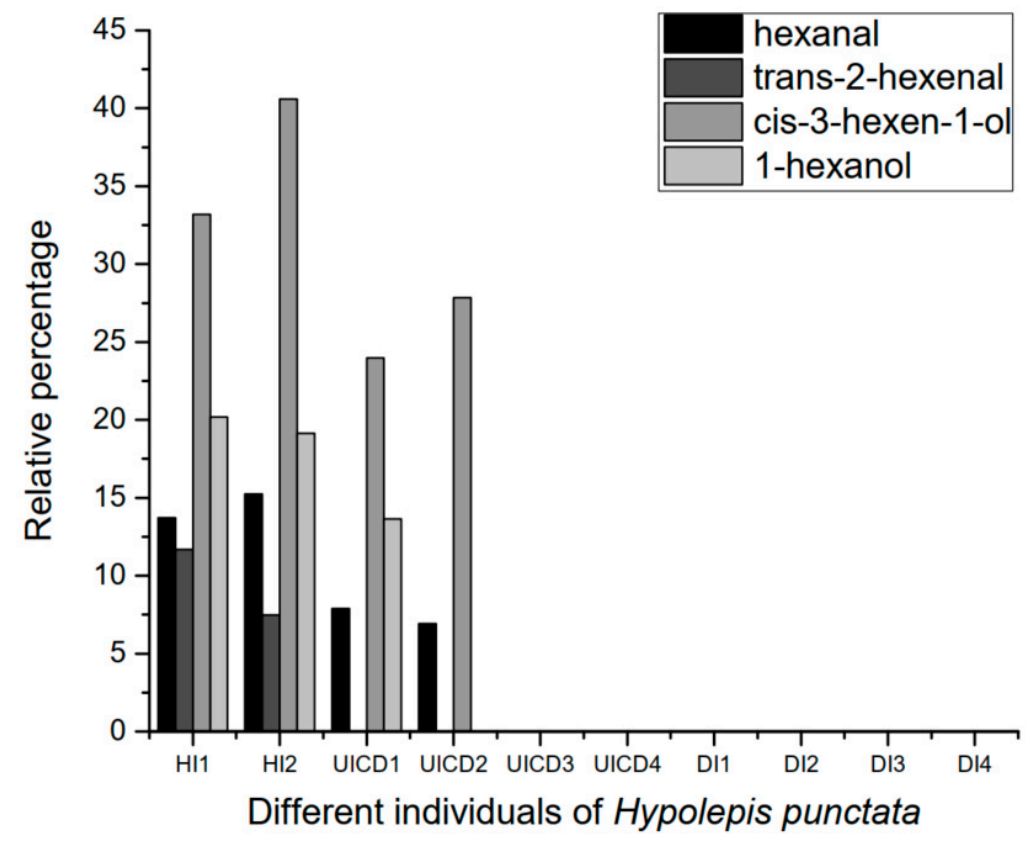

Figure 2. Relative percentages of amounts of four green leaf volatiles in each individual of Hypolepis punctata. The Y-axis is the relative percentage of four green leaf volatiles, of which each is represented with a unique color. The X-axis shows ten individuals of Hypolepis punctata. $\mathrm{HI}=$ healthy individuals, $\mathrm{UICD}=$ undamaged individuals that are close to damaged individuals, DI = damaged individuals. 


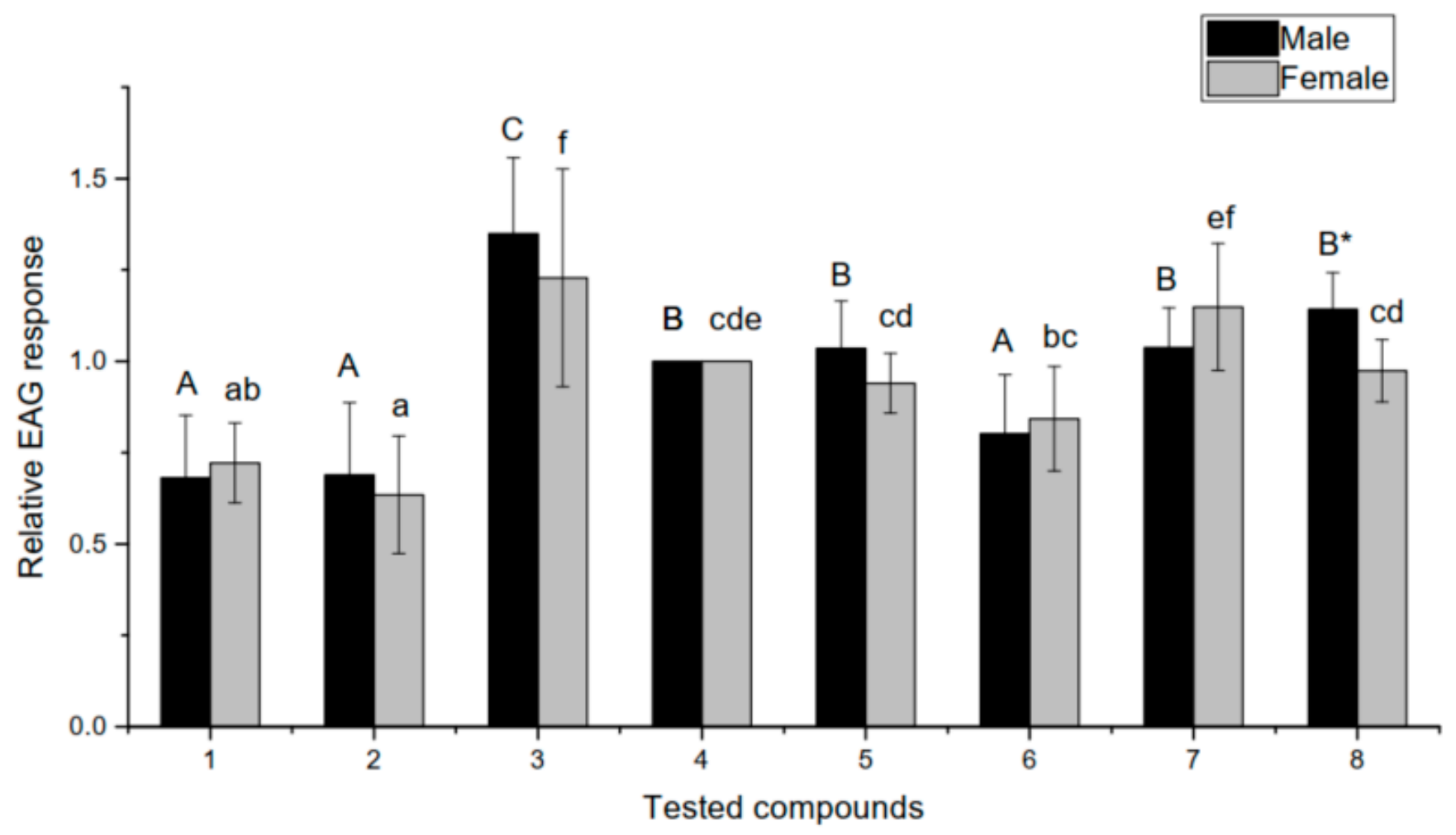

Figure 3. Relative EAG responses of Sclomina erinacea antenna to eight compounds. The Y-axis is the average relative EAG response of the antennae of Sclomina erinacea to eight selected compounds, which are represented by numbers $1-8$ on the $X$-axis: 1 = hexane, 2 = $\alpha$-pinene, 3 = trans-2-hexenal, $4=$ hexanal, $5=$ linalool, $6=\beta$-myrcene, $7=$ nonanal, $8=1$-octen-3-ol. Each insect sex is represented with a unique color, and the data was analyzed using Duncan's multiple range test within each sex between compounds. The uppercase or lowercase letters on top of each column are placed for the comparison of the EAG responses of males or females to each chemical, the uppercase letters are for males and the lowercase letters are for females. Columns with one or more common letters on top represent there is no significance $(p>0.05)$ between the EAG responses of antennae of one sex to each corresponding chemicals, otherwise the EAG responses to each corresponding chemicals for each column(s) with unique letter(s) is(are) significantly different from other chemicals. The symbol * means there is significant difference between the EAG responses of males and females to a chemical.

\subsection{Y-Olfactometer Experiment}

The number of choices of Sclomina erinacea among the seven tested compounds versus hexane using a Y-olfactometer was shown in Table 4, while the percent of choices is shown in Figure 4. To male S. erinacea, nonanal, $\beta$-myrcene, and trans-2-hexenal showed significant attractiveness $(p<0.05$, Table 4$)$. As to females, only linalool showed significant attraction $(p<0.05$, Table 4). However, although the difference was not significant, females prefer trans-2-hexenal (18 vs. 9 times, Table 4 ), and males prefer linalool (16 vs. 8 times, Table 4 ) than towards hexane. 
Table 4. Sclomina erinacea individuals' preference in Y-olfactometer experiment to seven kinds of VOCs produced by Hypolepis punctata versus hexane.

\begin{tabular}{|c|c|c|c|c|c|}
\hline Compounds & $\begin{array}{l}\text { Average Relative } \\
\text { Percent in DI Group } \\
(\%)\end{array}$ & Sex & $\begin{array}{l}\text { Number of Choices } \\
\text { to Experiment } \\
\text { Group }\end{array}$ & $\begin{array}{c}\text { Number of Choices } \\
\text { to Control (Hexane) } \\
\text { Group }\end{array}$ & $p$-Value \\
\hline \multirow{3}{*}{ linalool } & \multirow{3}{*}{20.63} & Total & 39 & 15 & *0.0013 \\
\hline & & Female & 23 & 7 & * 0.0027 \\
\hline & & Male & 16 & 8 & 0.0992 \\
\hline \multirow{3}{*}{$\beta$-myrcene } & \multirow{3}{*}{10.96} & Total & 30 & 18 & 0.0816 \\
\hline & & Female & 15 & 12 & 0.5633 \\
\hline & & Male & 15 & 6 & * 0.0459 \\
\hline \multirow{3}{*}{ alpha-pinene } & \multirow{3}{*}{4.85} & Total & 23 & 25 & 0.7728 \\
\hline & & Female & 14 & 13 & 0.8474 \\
\hline & & Male & 9 & 12 & 0.5120 \\
\hline \multirow{3}{*}{ hexanal } & \multirow{3}{*}{0.00} & Total & 20 & 28 & 0.2471 \\
\hline & & Female & 12 & 15 & 0.5633 \\
\hline & & Male & 8 & 13 & 0.2729 \\
\hline \multirow{3}{*}{ 1-octen-3-ol } & \multirow{3}{*}{4.48} & Total & 22 & 26 & 0.5635 \\
\hline & & Female & 8 & 13 & 0.2729 \\
\hline & & Male & 14 & 13 & 0.8474 \\
\hline \multirow{3}{*}{ trans-2-hexenal } & \multirow{3}{*}{0.00} & Total & 33 & 15 & $* 0.0085$ \\
\hline & & Female & 18 & 9 & 0.0803 \\
\hline & & Male & 15 & 6 & * 0.0459 \\
\hline \multirow{3}{*}{ nonanal } & \multirow{3}{*}{40.42} & Total & 26 & 22 & 0.5635 \\
\hline & & Female & 11 & 16 & 0.3345 \\
\hline & & Male & 15 & 6 & * 0.0459 \\
\hline
\end{tabular}

A G-test was used to compare the selection of different groups, and * indicates that there is a significant difference $(p<0.05)$ between the choices of the control group and the experimental group.

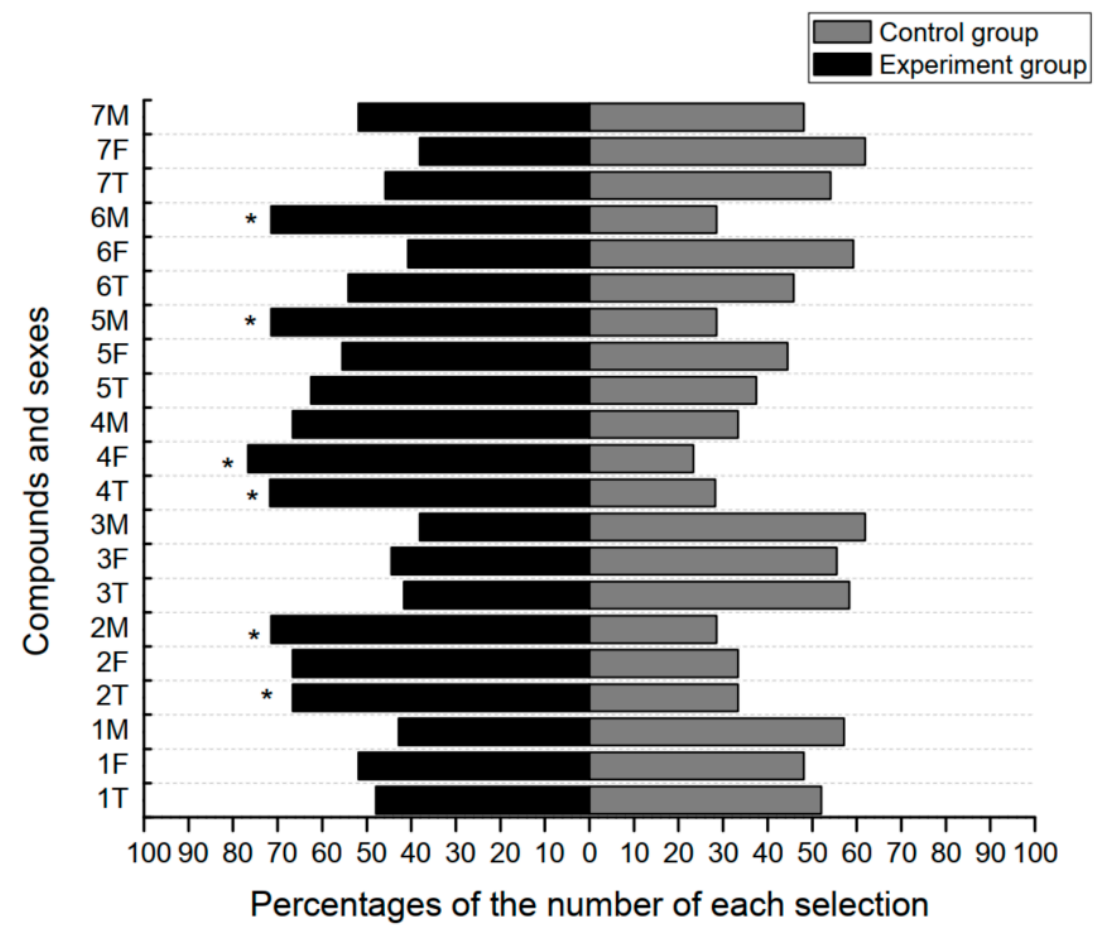

Figure 4. Sclomina erinacea individuals' preference to seven kinds of VOCs produced by Hypolepis punctata. Different numbers on the $Y$-axis represent seven different chemicals: $1=\alpha$-pinene; $2=$ trans-2-hexenal; $3=$ hexanal; $4=$ linalool; $5=$ $\beta$-myrcene; 6 = nonanal; 7 = 1-octen-3-ol; $\mathrm{M}=$ males; $\mathrm{F}=$ females; $\mathrm{T}=$ total. The proportion of the selection between the control group (hexane group) and the experimental group (different tested chemicals) in each condition on the Y-axis is represented using two different colors. A G-test was used to compare the selection of different groups, and * indicates that there is a significant difference between the choices of the control group and the experimental group. 


\section{Discussion}

Our study provides the first clear evidence of indirect defense mechanisms in ferns. Once wounded by herbivorous insects, $H$. punctata individuals were significantly more attractive to $S$. erinacea compared with healthy individuals in the field (Table 2). Furthermore, the damaged individuals produced a significant amount of novel volatiles compounds, which were not detected in healthy individuals of this fern species. Electroantennogram measurements and behavioral experiments using volatile standards indicated that these novel volatiles compounds attract $S$. erinacea.

\subsection{Volatiles Induced from $\mathrm{H}$. Punctata}

Our results illustrated that there was significantly more $\beta$-myrcene, linalool, nonanal, and marginally significantly more $\alpha$-pinene secreted from wounded $H$. punctata in comparison with intact individuals. The average amount of secreted 1-octene-3-ol was not significantly different between the $\mathrm{HI}$ and DI groups; however, the average amount of 1-octene3 -ol was higher from wounded than from healthy plants. Linalool [23], $\beta$-myrcene [24], $\alpha$-pinene [25], nonanal [26], and 1-octene-3-ol [27] have been shown to impact insect behavior. Among them, $\alpha$-pinene, linalool, and 1-octen-3-ol have been found to be able to attract predators for plant defense $[9,28,29]$. Therefore, combined the phenomenon with the result of our field observation, we believe that these induced secondary metabolites are volatiles produced in the fern, and they may play an essential role in the indirect defense of $H$. punctata by attracting natural enemies.

It is metabolically expensive for plants to synthesize induced volatiles, and sometimes the synthesis would result in a reduced or halted production of other common volatiles [21]. In this study, the four green leaf volatiles were detected in only healthy individuals but were not detected in or only detected in small amounts in wounded H. punctata or nearby healthy individuals. We speculate that to synthesize induced volatiles, H. punctata reduced the release of green leaf volatiles (Figure 4). This is a metabolic trade-off in the plant.

Plants can communicate via volatile chemicals. Studies have shown that when plants are injured, adjacent plants initiate the corresponding mechanism of chemical defense [10-12]. In this study, $\beta$-myrcene and linalool were found in healthy H. punctata individuals near wounded individuals. Wounded H. punctata likely communicated the wounding chemically with other individuals to induce them to produce volatiles. However, this hypothesis needs further exploration.

\subsection{Electrophysiological Experiments Using Volatile Standards}

Electrophysiological experiments were used to accurately determine whether the insects sense chemicals through smell, which is an excellent method to study the relationship between insects and volatiles. In particular, this allowed us to determine which compounds in the tested volatiles are likely to affect insect behavior [30]. Among the selected volatile standards, five were induced chemicals, and two were GLVs from healthy leaves. All volatiles except $\alpha$-pinene and $\beta$-myrcene generated stronger electrophysiological signals than the control, $\mathrm{n}$-hexane, in the antennae of $S$. erinacea, suggesting that S. erinacea may at least sense some of the induced substances produced in the fern and be attracted to assist the fern (the indirect defense mechanism). It is possible that not all volatiles produced by $H$. punctata can be sensed, which might be why two of the identified compounds did not significantly stimulate the antennae.

Additionally, there might be other unknown functions for these volatiles. Studies have shown that $\alpha$-pinene is a secondary metabolite with direct defense activity, which has been shown to reduce the number of larvae and adults Tribolium castaneum and decrease their food consumption and food utilization rate [31]. To our knowledge, there has been no report on the role of $\beta$-myrcene for effective defense in plants. In addition, it is possible that some of the induced components in our study may play a role in the chemical communication between plants of the same species because some of these induced chemical components were detected in individuals near the wounded plants. Green leaf volatiles (GLVs) are a 
kind of "common smell" emitted by many unwounded plants [32,33]. The releasing of Green leaf volatiles was not insect-wounding-needed. In our study, however, some of the GLVs (e.g., trans-2-hexenal) were able to generate electrophysiological signals in the antennae, and, notably, adult male and female $S$. erinacea were significantly more sensitive to trans-2-hexenal in EAG measurements $(p<0.05)$ than other compounds. This suggests that even before wounding, the insect may be able to sense the presence of H. punctata or plants with similar volatiles. This implies that $S$. erinacea may have adapted to sense non-induced plant scents to locate its habitat or proliferation site near potential hunting grounds, and then precisely locate its prey based on the induced chemical compounds, which requires further study.

In addition, there were no significant differences in the EAG measurements of other compounds between male and female S. erinacea, except for with 1-octen-3-ol, suggesting that the induced volatiles may not affect the reproductive behavior of S. erinacea, and the leading role of the volatiles is to attract the insects to catch pests.

\subsection{Behavioral Experiments Using Volatile Standards}

Compared with electrophysiological experiments, behavioral experiments can be easily influenced by environmental factors, especially when the tested compounds are unstable; the results are also influenced by the health of the tested insects. Furthermore, it is highly challenging to simulate the natural conditions of the olfactory condition entirely. Even so, among the seven tested volatiles, four had significantly stronger attractive activity on one sex of S. erinacea compared with the control, indicating that the induced volatile compounds attract $\mathrm{S}$. erinacea with more differential activity observed between sexes, especially when compared with the EAG experiments, which is also similarly reported in one other related study [34]. Further investigations are needed to have a better understanding of the behavioral patterns between male and female S. erinacea individuals.

Among the volatile compounds that attract $S$. erinacea, nonanal and 2-hexenal were from undamaged green leaves, and $\beta$-myrcene and linalool were induced after wounding. The attractive activity by the remaining three compounds (excluding $\beta$-myrcene) is in line with the results of the electrophysiological experiments, suggesting that the attractiveness of $H$. punctata to S. erinacea may be through an indirect defense mechanism. The fact that green leaf volatiles can attract $S$. erinacea also implies that the insect may establish its habitat based on constitutional (not induced) smells from $\mathrm{H}$. punctata. However, $\beta$ myrcene, which had a similar EAG value to hexane, was found to be attractive to adult male $S$. erinacea. This may be due to the reason that fewer odor sensilla that are sensitive to $\beta$-myrcene are in the antennae of the adult males than those sensilla sensitive to other compounds. Nonetheless, these sensilla still generate signals that impact the behavior of male S. erinacea. More studies are needed to elucidate the specific reasons for the attraction of males to $\beta$-myrcene. Our result also suggested that the attractiveness of these volatiles to $S$. erinacea seems to be irrelevant with their relative amounts (Table 4).

Our findings explain our preliminary field observations where we observed that noctuid larvae were heavily preyed on by S. erinacea when eating H. punctata. However, the effect of this defense mechanism is also dependent on the severity of the pest damage and the population density of S. erinacea. Therefore, further studies are needed to investigate the quantitative and dynamic relationship between noctuid larvae and S. erinacea.

\section{Conclusions}

Our data provide direct evidence that the herbivory-damaged H. punctata has more attractiveness to $S$. erinacea than the healthy individuals in the field, and the volatiles from $H$. punctata can attract $S$. erinacea, especially the induced compounds. This supports the proposition that ferns may also employ indirect defense mechanisms using volatile organic compounds. This mechanism is different from ants being attracted to fern species using nectar as a consumable reward [17] because H. punctata does not provide a direct reward to $S$. erinacea, yet still creates a mutually beneficial relationship with $S$. erinacea. Thus, 
we discovered an indirect defense mechanism of a fern species, and whether the volatile attraction mechanism found in this study resulted from long-term co-evolution still needs further research.

Supplementary Materials: The following are available online at https: / www.mdpi.com/article / 10.3390/insects12110978/s1, Figure S1: A sample graph of the whole device of the Y-olfactometer, Figure S2: A picture showing the position of an adult when it just reached the score point in the Y-olfactometer experiment.

Author Contributions: Conception and design of research, K.H., Y.Y., Q.Z. \& H.S. (Hui Shang); performed experiments, K.H., H.S. (Hui Shang) \& Q.Z.; analyzed data, K.H. \& H.S. (Hui Shang); interpreted results of experiments, K.H., Q.Z. and Y.Y.; First writing, K.H. and H.S. (Hui Shang); literature search, Y.W. and H.S. (Hui Shen); revised manuscript, K.H., Y.W., H.S. (Hui Shang) and H.S. (Hui Shen). All authors have read and agreed to the published version of the manuscript.

Funding: This research was financially supported by the Shanghai Municipal Administration of Forestation and City Appearances (Grant Nos. G212417, G192423, and F132421) and the National Natural Science Foundation of China (31700170).

Institutional Review Board Statement: Not applicable.

Informed Consent Statement: Not applicable.

Data Availability Statement: The datasets used during the current study are available from the corresponding author upon request.

Acknowledgments: The authors are grateful for the financial support from the Shanghai Municipal Administration of Forestation and City Appearances, and the National Natural Science Foundation of China. The authors wish to thank Tianbao Li (Hunan Normal University) and Chao Hu (Shanghai Chenshan Botanical Garden) for their support with the GC-MS device for VOCs sample analyses; Ling Zeng and Yajuan Xu (South China Agricultural University) for their support with devices used in electroantennogram recordings; Jiubing Zhang for his help with VOC sampling in the field; Yipeng Liu, Hongmei Liu, Shan Chen, Gang Li, Lu Liu and Harald Schneider for their good advice for the research program.

Conflicts of Interest: The authors declare that they have no conflict of interest.

$\begin{array}{ll}\text { Abbreviations } \\ \text { VOCs } & \text { Volatile organic compounds } \\ \text { EAG } & \text { electroantennogram } \\ \text { GLVs } & \text { green leaf volatiles }\end{array}$

\section{References}

1. Schoonhoven, L.M.; van Loon, J.J.; Dicke, M. Insect-Plant Biology; Oxford University Press: New York, NY, USA, 2005.

2. Gershenzon, J.; Dudareva, N. The function of terpene natural products in the natural world. Nat. Chem. Biol. 2007, 3, 408-414. [CrossRef] [PubMed]

3. Kaplan, I.; Halitschke, R.; Kessler, A.; Sardanelli, S.; Denno, R.F. Constitutive and induced defenses to herbivory in above- and belowground plant tissues. Ecology 2008, 89, 392-406. [CrossRef] [PubMed]

4. Mithöfer, A.; Boland, W. Plant defense against herbivores: Chemical aspects. Annu. Rev. Plant Biol. 2012, 63, 431-450. [CrossRef] [PubMed]

5. Duke, S.O.; Canel, C.; Rimando, A.M.; Tellez, M.R.; Duke, M.V.; Paul, R.N. Current and potential exploitation of plant glandular trichome productivity. Adv. Bot. Res. Inc. Adv. Plant Pathol. 2000, 31, 121-151.

6. Heil, M. Indirect defence via tritrophic interactions. New Phytol. 2007, 178, 41-61. [CrossRef]

7. Dicke, M.; van Beek, T.A.; Posthumus, M.A.; Ben-Dom, N.; van Bokhoven, H.; de Groot, A. Isolation and identification of volatile kairomone that affects acarine predatorprey interactions Involvement of host plant in its production. J. Chem. Ecol. 1990, 16, 381-396. [CrossRef] [PubMed]

8. de Moraes, C.M.; Lewis, W.J.; Pare, P.W.; Alborn, H.T.; Tumlinson, J.H. Herbivore-infested plants selectively attract parasitoids. Nature 1998, 393, 570-573. [CrossRef]

9. Kessler, A.; Baldwin, I.T. Defensive function of herbivore-induced plant volatile emissions in Nature. Science 2001, 291, 2141-2144. [CrossRef] 
10. Chessin, M.; Zipf, A.E. Alarm systems in higher plants. Bot. Rev. 1990, 56, 193-235. [CrossRef]

11. Karban, R.; Yang, L.; Edwards, K.F. Volatile communication between plants that affects herbivory: A meta-analysis. Ecol. Lett. 2013, 17, 44-52. [CrossRef]

12. Skoczek, A.; Piesik, D.; Wenda-Piesik, A.; Buszewski, B.; Bocianowski, J.; Wawrzyniak, M. Volatile organic compounds released by maize following herbivory or insect extract application and communication between plants. J. Appl. Èntomol. 2016, 141, 630-643. [CrossRef]

13. Weinhold, A.; Baldwin, I.T. Trichome-derived O-acyl sugars are a first meal for caterpillars that tags them for predation. Proc. Natl. Acad. Sci. USA 2011, 108, 7855-7859. [CrossRef] [PubMed]

14. Huang, S.-Q.; Zhang, Z.-X.; Li, Y.-Z.; Li, Y.-X.; Xu, H.-H. Anti-Insect activity of the methanol extracts of fern and gymnosperm. Agric. Sci. China 2010, 9, 249-256. [CrossRef]

15. Markham, K.; Chalk, T.; Stewart, C.N. Evaluation of fern and moss protein-based defenses against phytophagous insects. Int. J. Plant Sci. 2006, 167, 111-117. [CrossRef]

16. Imbiscuso, G.; Trotta, A.; Maffei, M.; Bossi, S. Herbivory induces a ROS burst and the release of volatile organic compounds in the fern Pteris vittata L. J. Plant Interact. 2009, 4, 15-22. [CrossRef]

17. Koptur, S.; Rico-Gray, V.; Palacios-Rios, M. Ant protection of the nectaried fern Polypodium plebeium in central Mexico. Am. J. Bot. 1998, 85, 736-739. [CrossRef]

18. McAuslane, H.J.; Metcalf, R.L.; Metcalf, E.R. Plant kairomones in insect ecology and control. Fla. Èntomol. 1992, 75, 613. [CrossRef]

19. Zhao, P.; Yuan, J.L. Theinsect list and faunal analysis of Harpactorinae in Guizhou province. Guizhou Agric. Sci. 2011, 37, 99-102.

20. Wu, Z.Y.; Raven, P.H.; Hong, D.Y. Flora of China; Lycopodiaceae through Polypodiaceae; Science Press: Beijing, China, 2013; Volume 2-3.

21. Paré, P.W.; Tumlinson, J.H. Plant volatiles as a defense against insect herbivores. Plant Physiol. 1999, 121, 325-332. [CrossRef]

22. Hayashi, Y.; Nishizawa, M.; Sakan, T. Studies on the sesquiterpenoids of Hypolepis punctata mett.-I: Isolation and structure determination of hypacrone, a new seco-illudoid. Tetrahedron 1977, 33, 2509-2511. [CrossRef]

23. Du, Y.; Poppy, G.M.; Powell, W.; Pickett, J.A.; Wadhams, L.J.; Woodcock, C.M. Identification of semiochemicals released during aphid feeding that attract parasitoid Aphidius ervi. J. Chem. Ecol. 1998, 24, 1355-1368. [CrossRef]

24. Ishkawa, M.; Shuto, Y.; Watanabe, H. $\beta$-Myrcene, a potent attractant component of pine wood for the pine wood nematode, Bursaphelenchus xylophilus. Agric. Biol. Chem. 1986, 50, 1863-1866. [CrossRef]

25. Schroeder, L.; Lindelöw, A. Attraction of scolytids and associated beetles by different absolute amounts and proportions of $\alpha$-pinene and ethanol. J. Chem. Ecol. 1989, 15, 807-817. [CrossRef]

26. Yu, H.; Zhang, Y.; Wu, K.; Gao, X.W.; Guo, Y.Y. Field-testing of synthetic herbivore-induced plant volatiles as attractants for beneficial insects. Environ. Èntomol. 2008, 37, 1410-1415. [CrossRef]

27. Takken, W.; Kline, D. Carbon dioxide and 1-octen-3-ol as mosquito attractants. J. Am. Mosq. Control Assoc. 1989, 5, 311-316. [PubMed]

28. Zhu, J.; Cossé, A.A.; Obrycki, J.J.; Boo, K.S.; Baker, T.C. Olfactory reactions of the twelve-spotted lady beetle, Coleomegilla maculata and the green lacewing, Chrysoperla carnea to semiochemicals released from their prey and host plant: Electroantennogram and behavioral responses. J. Chem. Ecol. 1999, 25, 1163-1177. [CrossRef]

29. Erbilgin, N.; Raffa, K.F. Modulation of predator attraction to pheromones of two prey species by stereochemistry of plant volatiles. Oecologia 2001, 127, 444-453. [CrossRef] [PubMed]

30. Guerin, P.M.; Visser, J.H. Electroantennogram responses of the carrot fly, Psila rosae, to volatile plant components. Physiol. Èntomol. 1980, 5, 111-119. [CrossRef]

31. Huang, Y.; Hee, S.; Ho, S. Antifeedant and growth inhibitory effects of $\alpha$-pinene on the stored-product insects, Tribolium castaneum (Herbst) and Sitophilus zeamais Motsch. Int. Pest Control 1998, 40, 18-20.

32. Hoballah, M.E.F.; Tamò, C.; Turlings, T.C. Differential attractiveness of induced odors emitted by eight maize varieties for the parasitoid Cotesia marginiventris: Is quality or quantity important? J. Chem. Ecol. 2002, 28, 951-968. [CrossRef] [PubMed]

33. Piesik, D.; Rochat, D.; Delaney, K.; Marion-Poll, F. Orientation of European corn borer first instar larvae to synthetic green leaf volatiles. J. Appl. Entomol. 2013, 137, 234-240. [CrossRef]

34. Piesik, D.; Wenda-Piesik, A. Sitophilus granarius responses to blends of five groups of cereal kernels and one group of plant volatiles. J. Stored Prod. Res. 2015, 62, 36-39. [CrossRef] 